\title{
2-Cyanomethylbenzaldehyde - useful substrate for preparation of some 1,3-di- and 1,2,3-trisubstituted naphthalenes or substituted 1-cyanobenzobicyclo[2.2.2]octenes
}

\author{
Marek Panasiewicz, Tadeusz Zdrojewski, Krzysztof Chrulski, Anna Wojtasiewicz, \\ and Andrzej Jończyk*
}

Warsaw University of Technology, Faculty of Chemistry

Noakowskiego St. 3, 00-664 Warsaw, Poland

E-mail: anjon@ch.pw.edu.pl

\begin{abstract}
2-Cyanomethylbenzaldehyde reacts in the presence of concentrated aqueous solution of sodium hydroxide or powdered potassium carbonate and a quaternary ammonium salt as a catalyst in benzene (phase-transfer catalysis) with 2-chloroethyl aryl ketones or electrophilic alkenes affording 1-cyano-3-acylnaphthalenes, 1-(2-cyano)- and 1-(2-phenylsulfonyl)ethyl-3-substituted naphthalenes or 1-cyano-5,8-diaroyl-benzobicyclo[2.2.2] octenes. Further cyclization of the corresponding 5,8-diacetyl derivative of 1-cyanobenzobicyclooctene gives 1-cyano-10methyltetracyclo[7.5.1. $\left.0^{2,7} \cdot 0^{8,13}\right]$ pentadeca-2,4,6,10-tetraen-12-one. Formation of these products is rationalized.
\end{abstract}

Keywords: Phase-transfer catalysis, Michael reaction, annulation, cyclization, carbocycles, redox reaction

\section{Introduction}

The cyanomethyl group together with the adjoining formyl group are suitably arranged in a molecule of 2-cyanomethylbenzaldehyde (1) ${ }^{1}$ to participate in the formation of fused-ring compounds. Indeed, the reaction of aldehyde 1 with ammonia, primary or secondary amines, catalyzed by trifluoroacetic acid, gives 3-amino substituted isoquinolines, usually in good yields. $^{2}$ In this case, amidines formed by the addition of ammonia or amines to the cyano group in 1 undergo cyclization with the formyl group. We expected that in an analogous process, anions $4^{-}$generated in Michael reaction of $\mathbf{1}^{-}$with electrophilic alkenes 3 (where EWG is an electron withdrawing group), should be intercepted by the formyl group forming dihydronaphthalene derivatives 5 . 


\section{Results and Discussion}

However, the reaction of $\mathbf{1}$ with equimolar amounts (or slight excess) of 2-chloroethyl aryl ketones 2, aryl or alkyl vinyl ketones 3, carried out under phase-transfer catalysis conditions, $\left(\mathrm{PTC}^{3-6}\right)$, i.e. in the presence of $50 \%$ aqueous sodium hydroxide and benzyltriethylammonium chloride (TEBAC) as a catalyst (system A) or powdered potassium carbonate and Aliquat 336 as a catalyst (system B), afforded 1-cyano-3-acylnaphthalenes 6a-g, in 53-73\% yield (Scheme 1, Table 1).
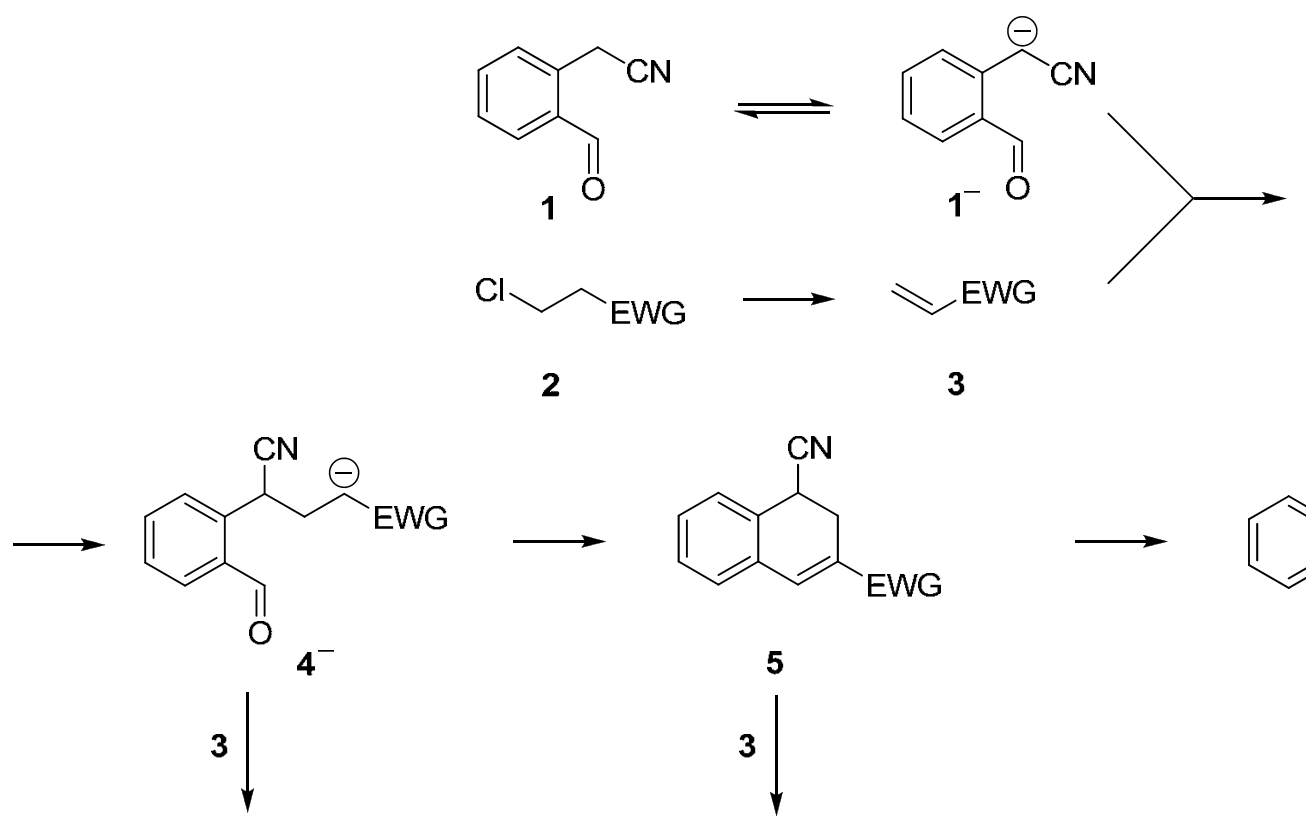<smiles>CC1=Cc2ccccc2C(C#N)C1</smiles><smiles>CCc1cc(C#N)c2ccccc2c1</smiles><smiles>CC(C)CCC(C#N)(CCC(C)(C)C)c1ccccc1C=O</smiles>

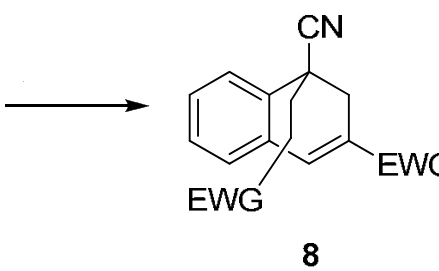<smiles></smiles><smiles>CC(C)(C)CCc1cc(C(C)(C)C)cc2ccccc12</smiles>

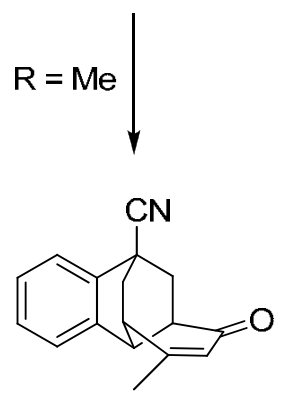

Scheme 1. Formation of fused-ring products 6, 9, 10 and 11. 
Table 1. Products 6, 9 and 10 prepared

\begin{tabular}{|c|c|c|c|c|c|c|}
\hline \multirow[t]{2}{*}{ No. } & \multirow[t]{2}{*}{2 or $\mathbf{3}$; EWG } & \multirow[t]{2}{*}{ System $^{\mathrm{a}}$} & \multirow{2}{*}{$\begin{array}{l}(\mathbf{2} \text { or } \mathbf{3}) / \mathbf{1} \\
(\mathrm{mol} / \mathrm{mol})\end{array}$} & \multicolumn{3}{|c|}{ Yield [\%] } \\
\hline & & & & 6 & 9 & 10 \\
\hline \multirow[t]{3}{*}{1} & $\mathbf{2 a} ; \mathrm{C}_{6} \mathrm{H}_{5} \mathrm{CO}$ & A & 1.0 & 68 & - & - \\
\hline & & & 2.1 & 5 & 60 & - \\
\hline & $\mathbf{3 a} ; \mathrm{C}_{6} \mathrm{H}_{5} \mathrm{CO}$ & A & 1.0 & $41^{\mathrm{b}}$ & - & - \\
\hline \multirow[t]{2}{*}{2} & $\mathbf{2 b} ; 4-\mathrm{MeC}_{6} \mathrm{H}_{4} \mathrm{CO}$ & A & 1.0 & 53 & - & - \\
\hline & & & 2.1 & - & 52 & - \\
\hline \multirow[t]{2}{*}{3} & $\mathbf{2 c} ; 4-\mathrm{ClC}_{6} \mathrm{H}_{4} \mathrm{CO}$ & A & 1.0 & 55 & - & - \\
\hline & & & 2.1 & - & 78 & - \\
\hline \multirow[t]{2}{*}{4} & 2d; $4-\mathrm{BrC}_{6} \mathrm{H}_{4} \mathrm{CO}$ & A & 1.0 & 73 & - & - \\
\hline & & & 2.1 & - & 50 & - \\
\hline \multirow[t]{2}{*}{5} & $\mathbf{2 e} ; 3,4-(\mathrm{MeO})_{2} \mathrm{C}_{6} \mathrm{H}_{3} \mathrm{CO}$ & A & 1.0 & 54 & - & - \\
\hline & & & 2.1 & 5 & 47 & - \\
\hline \multirow[t]{2}{*}{6} & 3f; $\mathrm{CH}_{3} \mathrm{CO}$ & A & 1.0 & $11^{\mathrm{b}}$ & - & - \\
\hline & & B & 1.0 & 72 & - & - \\
\hline \multirow[t]{2}{*}{7} & 3g; $\mathrm{C}_{2} \mathrm{H}_{5} \mathrm{CO}$ & A & 1.0 & $38^{\mathrm{b}}$ & - & - \\
\hline & & B & 1.0 & 60 & - & - \\
\hline 8 & $3 \mathbf{h} ; \mathrm{CN}$ & A & 2.2 & - & - & 34 \\
\hline 9 & $3 \mathbf{i} ; \mathrm{SO}_{2} \mathrm{Ph}$ & A & 2.2 & - & - & 78 \\
\hline
\end{tabular}

${ }^{\mathrm{a}} \mathrm{A}: 50 \%$ aq. $\mathrm{NaOH}$, cat. TEBAC; B: powd. $\mathrm{K}_{2} \mathrm{CO}_{3}$, cat. Aliquat $336 .{ }^{\mathrm{b}} \mathrm{A}$ lot of tars were formed.

The reaction of 1 with one equivalent of phenyl vinyl ketone 3a $(E W G=B z)$ afforded naphthalene 6a, however in a lower yield comparing to the one where ketone 2a was used (Table 1; No.1). Aryl vinyl ketones are unstable, easily decomposed when treated with a base, hence the use of their precursors, ketones 2, in more basic System A is advisable.

Structurally related products $\mathbf{1 3}$ were also formed from the reaction of $\mathbf{1}$ with cyclic ketones 12a,b (used in significant excess). In this case the best results gave the solid-liquid PTC system B (Scheme 2).<smiles>N#CCc1ccccc1C=O</smiles>

1<smiles>O=C1C=CC2CCCCC12</smiles>

12<smiles>N#Cc1c2c(cc3ccccc13)C(=O)CC2</smiles>

$\begin{array}{ll}\text { a, } n=1 & 55 \% \\ \text { b, } n=2 & 80 \%\end{array}$

Scheme 2. Reaction of aldehyde $\mathbf{1}$ with cyclic ketones 12. 
Products $\mathbf{6}$ and $\mathbf{1 3}$ are not described in the literature. Synthesis of naphthalenes substituted at C-1 and C-3 with EWG-s is not easily realized, and such products were often obtained in low yields, usually by processes other than aromatic electrophilic substitution (e.g. 1,3-dinitro-, ${ }^{7,8}$ 1,3-dicyano-, ${ }^{9} \quad$ 1-nitro-3-acyl-, ${ }^{10} \quad 3$-cyano-1-methoxycarbonyl- ${ }^{11}$ or 1-cyano-3methoxycarbonylnaphthalene ${ }^{12}$ ). Recently, the synthesis and transformations of fused bicyclo[2.2.2] octenes have been reviewed. ${ }^{13}$

A possible route leading to products $\mathbf{6}$ is presented on Scheme 1. Carbanion $\mathbf{1}^{-}$generated from $\mathbf{1}$ reacted with ketone $\mathbf{2}$ or $\mathbf{3}$ (in the case of chloroketones $\mathbf{2}$ most likely via aryl vinyl ketone 3), forming anion $4^{-}$. Intramolecular aldol condensation of the latter followed by the elimination of water produces dihydronaphthalene $\mathbf{5}$, which is dehydrogenated to the final naphthalene 6. Conversion of 5 into $\mathbf{6}$ (Scheme 1) requires comments. It is well established that PTC oxidation of $\alpha$-arylalkanenitriles with dioxygen leads to the formation of corresponding phenones. ${ }^{14-16}$ In the case of $\mathbf{5}$, this reaction should give 3-aroyl-1-naphthols (by an oxidationtautomerization route), which in fact were not formed. On the other hand, 2-substituted derivatives of 1,4-dicyano-1,2-dihydronaphthalene treated with 5\% ethanolic potassium hydroxide were dehydrogenated into 1,4-dicyanonaphthalenes in low yield, but aromatization comprised mainly elimination of hydrogen cyanide. ${ }^{17}$ Partially unsaturated, cyano-substituted fused aromatic compounds were dehydrogenated with DDQ. ${ }^{18-20}$

Therefore, we undertook the independent synthesis of 5a (Scheme 3) to investigate its transformation into $\mathbf{6 a}$.<smiles>N#CCc1ccccc1C=O</smiles>

1<smiles>N#CC(CCC(=O)c1ccccc1)c1ccccc1C1OCCO1</smiles>

15<smiles>N#CC1CC(C(=O)c2ccccc2)=Cc2ccccc21</smiles>

$5 a$

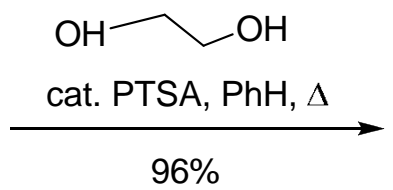

$78 \%$

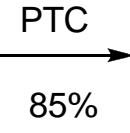

$85 \%$

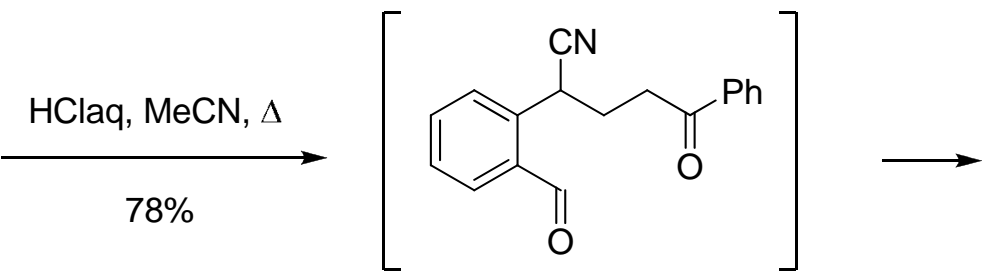<smiles>N#CCc1ccccc1C1OCCO1</smiles>

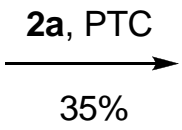

14

$16 a$<smiles>N#Cc1cc(C(=O)c2ccccc2)cc2ccccc12</smiles>

$6 a$

Scheme 3. Independent synthesis of $5 \mathbf{a}$ and its transformation into $6 \mathbf{a}$ in PTC system A. 
Acetalization of 1 gave quantitatively $\mathbf{1 4}$ which under PTC conditions reacted sluggishly with ketone $\mathbf{2 a}$ affording the Michael adduct $\mathbf{1 5}$. The reaction progress monitored by GC revealed total conversion of 14, but column chromatography allowed isolation of $\mathbf{1 5}$ in ca. 35\% yield, at best. Attempted deacetalization of $\mathbf{1 5}$ with hydrochloric acid in acetonitrile led directly to $\mathbf{5 a}$, instead of $\mathbf{1 6 a}$.

Next, the key intermediate 5a was stirred in the PTC system under air, under inert gas or in the stream of bubbled oxygen. Under the two first conditions, the process led to product 6a, but at a much higher rate under atmosphere of air, while passing oxygen into the reaction gave a complex mixture of products. The results testify to the spontaneous character of $\mathbf{5}$ into $\mathbf{6}$ transformation, difficult to control. Interestingly, we did not observe the formation of a dehydrocyanation product, i.e. 2-benzoylnaphthalene.

The reaction of methylene compounds with electrophilic alkenes often led to substitution of both acidic hydrogen atoms, particularly when an excess of the anion acceptor was applied. ${ }^{21-23}$ A similar process, additionally involving the formyl group in 1, was observed. Thus, stirring of 1 with more than two molar equivalents of ketones 2 produced tricyclic derivatives 9 in a 47-78\% yield (Scheme 1, Table 1). The reaction of 1 with an excess of methyl vinyl ketone (3a) gave intermediate 9 which possesses an active methyl group hence it reacted further giving polycyclic products 11.

Concerning tricyclic structure 9, the parent hydrocarbon, i.e. benzobicyclo[2.2.2]octane ${ }^{24-28}$ and some of its derivatives (e.g. 1-hydroxy-, ${ }^{24,29,30} 1$-methoxy-, ${ }^{25,30,31} 1$-chloro-, ${ }^{25,29} 1$-bromo- and 1 -acetoxy-, ${ }^{25}$ 2-cyano-, ${ }^{32,33} 2,3$-dimethoxycarbonyl- ${ }^{32}$ or 2,7 -dibromo- ${ }^{34}$ ) were prepared by multistep procedures, usually in low yields, while compounds listed in Table 1 are not described in the literature.

When an excess of ketone 2 was present in the system, the $\alpha$-cyanobenzyl carbanion generated from $4^{-}$via $[1,3]$ hydrogen shift reacted with the vinyl ketone 3 to give the anion of diadduct $7^{-}$, which via a series of intramolecular reactions afforded the final structure 9 (Scheme 1). Alternatively, intermediate diketone $\mathbf{8}$ may be also produced by the reaction of an anion from cyanodihydronaphthalene $\mathbf{5}$ with vinyl ketone $\mathbf{3}$.

Compounds 9a,c-e were isolated as pure diastereoisomers, possessing the same relative configuration at the carbon atoms bearing the aroyl groups (A and its mirror image; Figure 1). Their structure was fully confirmed by ${ }^{13} \mathrm{C}$ NMR spectra (two signals of carbonyl carbons). Surprisingly, the ${ }^{13} \mathrm{C}$ NMR spectrum of the product of the reaction of aldehyde $\mathbf{1}$ with ketone $\mathbf{2 b}$ confirms the identically arranged aroyl groups in $\mathbf{9 b}$ (diastereoisomer $\mathbf{B}$ or $\mathbf{C}$; Figure 1). Attempted equilibration of product $\mathbf{9 b}$ (stirring with 50\% aq. sodium hydroxide in benzene with TEBAC catalyst for $2 \mathrm{~h}$ ) failed since it decomposed. 


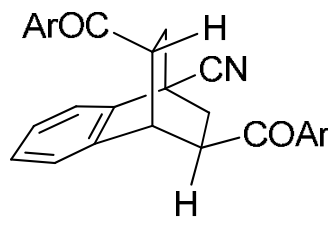

A

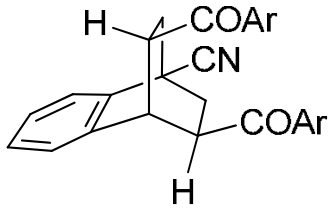

B

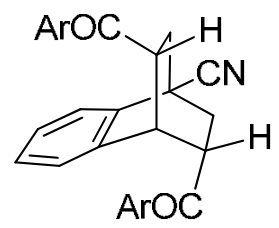

C

Figure 1. Possible orientation of aroyl groups in compounds 9.

Still other products were formed from $\mathbf{1}$ and electrophlic alkenes $\mathbf{3 h}$,i. In these cases, anions from 8 were not prone to cyclize but eliminated hydrogen cyanide giving naphthalene derivatives 10h,i.

\section{Conclusions}

We have described a simple approach to derivatives of cyanonaphthalenes $\mathbf{6}$ and cyanobenzobicyclo[2,2,2]octenes $\mathbf{9}$ from available aldehyde $\mathbf{1}$ and ketones $\mathbf{2}$ or $\mathbf{3}$, under convenient PTC conditions. Furthermore, we have indicated that suitably substituted 1-cyano1,2-dihydronaphthalenes 5 entered under PTC conditions spontaneous dehydrogenation into 1cyanonaphthalene derivatives $\mathbf{6}$. The intermediate tricyclic structure $\mathbf{9 f}$ [produced from $\mathbf{1}$ and methyl vinyl ketone (3f)] reacted further giving the fused-ring product 11. On the other hand, the PTC reaction of $\mathbf{1}$ with electrophilic alkenes $\mathbf{3 h}, \mathbf{i}$ afforded 1,3-disubstituted naphthalenes $\mathbf{1 0 h , i}$. The formation of these products is rationalized.

\section{Experimental Section}

General Procedures. Column chromatography was performed on Merck silica gel $(240 \div 400$ mesh) using AcOEt-hexane (gradient) as eluent, and gas chromatography (GC) on an Agilent 6850 Series GC System fitted with HP-50+ $(30 \mathrm{~m})$ column. ${ }^{1} \mathrm{H} \mathrm{NMR}\left(400 \mathrm{MHz}, \mathrm{CDCl}_{3}\right)$ and ${ }^{13} \mathrm{C}$ NMR (100 MHz, $\mathrm{CDCl}_{3}$ ) spectra were measured on a Varian Mercury 400BB spectrometer, if not indicated otherwise; chemical shifts $(\delta)$ are given in ppm related to tetramethylsilane (TMS), coupling constants $J$ in Hz. IR spectra were recorded on a Specord M-80 spectrophotometer in $\mathrm{KBr}$; $v$ are given in $\mathrm{cm}^{-1}$. Elemental analyses were performed on a Perkin Elmer 2400 Ser. II $\mathrm{CHNS} / \mathrm{O}$ microanalyser. Melting points were measured on a capillary apparatus, and were not corrected.

Aldehyde $\mathbf{1},{ }^{1}$ chloroethyl aryl ketones $\mathbf{2} \mathbf{a}-\mathbf{d}^{35}$ and $\mathbf{2 \mathbf { e } ^ { 3 6 }}$ were obtained according to literature procedures. 
General procedure for preparation of 1-cyano-3-aroylnaphthalenes 6a-e in system A

To the stirred solution of aldehyde $1(0.29 \mathrm{~g}, 2.0 \mathrm{mmol})$, chloroketone $\mathbf{2 a}-\mathbf{e}(2.0 \mathrm{mmol})$ and TEBAC $(0.023 \mathrm{~g}, 0.1 \mathrm{mmol})$ in benzene $(30 \mathrm{~mL}), 50 \% \mathrm{aq}_{\mathrm{aq}} \mathrm{NaOH}(1 \mathrm{~mL}, 19.1 \mathrm{mmol})$ was added. When aldehyde 1 was no longer detected by GC (1-2 h), the reaction mixture was diluted with water $(50 \mathrm{~mL})$. The organic phase was separated and the water phase was extracted with $\mathrm{CHCl}_{3}$ $(3 \times 20 \mathrm{~mL})$. Combined organic phases were washed with water $(30 \mathrm{~mL})$ and dried $\left(\mathrm{MgSO}_{4}\right)$. After the solvent was evaporated, the crude mixture was purified by column chromatography (Table 1). The products were recrystallized to give analytical samples of 6a-e.

1-Cyano-3-benzoylnaphthalene (6a). Mp 155-156 ${ }^{\circ} \mathrm{C}(\mathrm{MeOH})$. Yield: $350 \mathrm{mg}(68 \%)$. ${ }^{1} \mathrm{H}$ NMR: $\delta=7.53-7.57\left(\mathrm{~m}, 2 \mathrm{H}, \mathrm{CH}_{\mathrm{Ar}}\right)$, 7.65-7.69 (m, $\left.2 \mathrm{H}, \mathrm{CH}_{\mathrm{Ar}}\right), 7.70-7.74\left(\mathrm{~m}, 1 \mathrm{H}, \mathrm{CH}_{\mathrm{Ar}}\right)$, 7.82$7.86\left(\mathrm{~m}, 2 \mathrm{H}, \mathrm{CH}_{\mathrm{Ar}}\right), 8.03\left(\mathrm{~d}, J=8.0,1 \mathrm{H}, \mathrm{CH}_{\mathrm{Ar}}\right), 8.31\left(\mathrm{dd}, J=8.0, J=1.6,1 \mathrm{H}, \mathrm{CH}_{\mathrm{Ar}}\right), 8.37$ (d, $\left.J=1.6,1 \mathrm{H}, \mathrm{CH}_{\mathrm{Ar}}\right), 8.50\left(\mathrm{~s}, 1 \mathrm{H}, \mathrm{CH}_{\mathrm{Ar}}\right) \mathrm{ppm} .{ }^{13} \mathrm{C} \mathrm{NMR}: \delta=111.0,117.0,125.2,128.5,128.7$, $130.0,130.9,132.0,132.6,133.1,133.7,134.1,135.9,136.7,194.4$ ppm. IR: $v=3060,2224$, 1640, 1596, 1300, 916, $716 \mathrm{~cm}^{-1} . \mathrm{C}_{18} \mathrm{H}_{11} \mathrm{NO}$ (257.3): calcd. C 84.03, H 4.31, N 5.44; found: C 83.89, H 4.28, N 5.36.

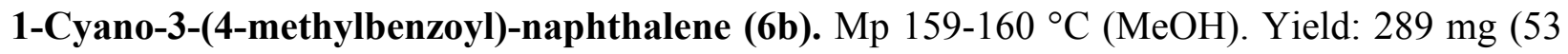
\%). ${ }^{1} \mathrm{H}$ NMR: $\delta=2.49$ (s, $\left.3 \mathrm{H}, \mathrm{CH}_{3}\right), 7.35\left(\mathrm{~d}, J=8.0,2 \mathrm{H}, \mathrm{CH}_{\mathrm{Ar}}\right), 7.70-7.76\left(\mathrm{~m}, 3 \mathrm{H}, \mathrm{CH}_{\mathrm{Ar}}\right.$ ), 7.82-7.86 (m, $\left.1 \mathrm{H}, \mathrm{CH}_{\mathrm{Ar}}\right), 8.03\left(\mathrm{~d}, J=8.0,1 \mathrm{H}, \mathrm{CH}_{\mathrm{Ar}}\right), 8.32\left(\mathrm{~d}, J=8.0,1 \mathrm{H}, \mathrm{CH}_{\mathrm{Ar}}\right), 8.36(\mathrm{~d}, J=$ 1.6, $\left.1 \mathrm{H}, \mathrm{CH}_{\mathrm{Ar}}\right), 8.49\left(\mathrm{~s}, 1 \mathrm{H}, \mathrm{CH}_{\mathrm{Ar}}\right) \mathrm{ppm} .{ }^{13} \mathrm{C} \mathrm{NMR}: \delta=21.8,110.8,117.1,125.3,128.5$, $129.4,130.1,130.2,130.8,132.0,132.7,133.6,134.0,134.5,135.8,144.1,194.2$ ppm. IR: $v=$ 3068, 2220, 1644, 1604, 1292, $764 \mathrm{~cm}^{-1}$. $\mathrm{C}_{19} \mathrm{H}_{13} \mathrm{NO}$ (271.3): calcd. C 84.11, H 4.83, N 5.16; found: C 83,85, H 4.70, N 5.13.

1-Cyano-3-(4-chlorobenzoyl)-naphthalene (6c). $\mathrm{Mp} 137-138{ }^{\circ} \mathrm{C}(\mathrm{MeOH})$. Yield: $321 \mathrm{mg}(55$ \%). ${ }^{1} \mathrm{H}$ NMR: $\delta=7.52-7.55\left(\mathrm{~m}, 2 \mathrm{H}, \mathrm{CH}_{\mathrm{Ar}}\right), 7.71-7.76\left(\mathrm{~m}, 1 \mathrm{H}, \mathrm{CH}_{\mathrm{Ar}}\right), 7.78-7.81\left(\mathrm{~m}, 2 \mathrm{H}, \mathrm{CH}_{\mathrm{Ar}}\right)$, 7.84-7.88 (m, $\left.1 \mathrm{H}, \mathrm{CH}_{\mathrm{Ar}}\right), 8.03\left(\mathrm{~d}, J=8.4,1 \mathrm{H}, \mathrm{CH}_{\mathrm{Ar}}\right), 8.33\left(\mathrm{dd}, J=8.4, J=1.6,1 \mathrm{H}, \mathrm{CH}_{\mathrm{Ar}}\right.$ ), $8.35\left(\mathrm{~d}, J=1.6,1 \mathrm{H}, \mathrm{CH}_{\mathrm{Ar}}\right), 8.47\left(\mathrm{~s}, 1 \mathrm{H}, \mathrm{CH}_{\mathrm{Ar}}\right) \mathrm{ppm} .{ }^{13} \mathrm{C} \mathrm{NMR}: \delta=111.1,116.9,125.2,128,8$, $129.0,130,1,131,2,131.3,131.9,132.3,133.6,133.7,134.9,135.8,139.6,193.2$ ppm. IR: $v=$ $3068,2224,1652,1584,1300,1092,768 \mathrm{~cm}^{-1} . \mathrm{C}_{18} \mathrm{H}_{10} \mathrm{ClNO}$ (291.7): calcd. C 74.11, H 3.45, N 4.80, Cl 12.15; found: C 74.13, H 3.25, N 4.78, Cl 12.08.

1-Cyano-3-(4-bromobenzoyl)-naphthalene (6d). Mp 131-132 ${ }^{\circ} \mathrm{C}(\mathrm{MeOH})$. Yield: $491 \mathrm{mg}(73$ \%). ${ }^{1} \mathrm{H}$ NMR: $\delta=7.66-7.75\left(\mathrm{~m}, 5 \mathrm{H}, \mathrm{CH}_{\mathrm{Ar}}\right), 7.83-7.87\left(\mathrm{~m}, 1 \mathrm{H}, \mathrm{CH}_{\mathrm{Ar}}\right), 8.03(\mathrm{~d}, J=8.4,1 \mathrm{H}$, $\left.\mathrm{CH}_{\mathrm{Ar}}\right), 8.31\left(\mathrm{~d}, J=8.4,1 \mathrm{H}, \mathrm{CH}_{\mathrm{Ar}}\right), 8.34\left(\mathrm{~d}, J=1.6,1 \mathrm{H}, \mathrm{CH}_{\mathrm{Ar}}\right), 8.46\left(\mathrm{~s}, 1 \mathrm{H}, \mathrm{CH}_{\mathrm{Ar}}\right) \mathrm{ppm} .{ }^{13} \mathrm{C}$ NMR: $\delta=111.1,116.9,125.2,128.2,128.6,130.1,131.0,131.4,131.9,132.0,132.3,133.60$, 133.62, 135.3, 135.8, 193.3 ppm. IR: $v=3064,2224,1652,1584,1292,1068,764 \mathrm{~cm}^{-1}$. $\mathrm{C}_{18} \mathrm{H}_{10} \mathrm{BrNO}$ (336.2): calcd. C 64.31, H 3.00, N 4.17, Br 23.77; found: C 64.47, H 2.82, N 3.99, Br 23.69.

1-Cyano-3-(3,4-dimethoxybenzoyl)-naphthalene (6e). Mp 169-170 ${ }^{\circ} \mathrm{C}(\mathrm{MeOH})$. Yield: 343 mg (54\%). ${ }^{1} \mathrm{H}$ NMR: $\delta=3.89\left(\mathrm{~s}, 3 \mathrm{H}, \mathrm{OCH}_{3}\right), 3.93\left(\mathrm{~s}, 3 \mathrm{H}, \mathrm{OCH}_{3}\right), 6.88\left(\mathrm{~d}, J=8.4,1 \mathrm{H}, \mathrm{CH}_{\mathrm{Ar}}\right)$, $7.32\left(\mathrm{dd}, J=8.4, J=1.6,1 \mathrm{H}, \mathrm{CH}_{\mathrm{Ar}}\right), 7.46\left(\mathrm{~d}, J=1.6,1 \mathrm{H}, \mathrm{CH}_{\mathrm{Ar}}\right), 7.63-7.69\left(\mathrm{~m}, 1 \mathrm{H}, \mathrm{CH}_{\mathrm{Ar}}\right)$, 7.73-7.75 (m, $\left.1 \mathrm{H}, \mathrm{CH}_{\mathrm{Ar}}\right), 7.97\left(\mathrm{~d}, J=8.4,1 \mathrm{H}, \mathrm{CH}_{\mathrm{Ar}}\right), 8.19\left(\mathrm{~d}, J=8.4,1 \mathrm{H}, \mathrm{CH}_{\mathrm{Ar}}\right), 8.22(\mathrm{~d}, J=$ 
1.6, $\left.1 \mathrm{H}, \mathrm{CH}_{\mathrm{Ar}}\right), 8.40\left(\mathrm{~s}, 1 \mathrm{H}, \mathrm{CH}_{\mathrm{Ar}}\right) \mathrm{ppm} .{ }^{13} \mathrm{C} \mathrm{NMR:} \delta=55.9,56.0,109.7,110.4,111.7,116.9$, $124.9,125.3,128.2,129.1,129.8,130.4,131.7,132.4,133.1,134.5,149.1,153.3,192.8$, ppm. IR: $v=3084,2964,2840,2236,1664,1512,1260,1152,1020,768 \mathrm{~cm}^{-1} . \mathrm{C}_{20} \mathrm{H}_{15} \mathrm{NO}_{3}(317.3)$ : calcd. C 75.70, H 4.76, N 4.41; found: C 75.56, H 4.81, N 4.32.

\section{General procedure for preparation of 1-cyano-3-acylnaphthalenes 6 f,g in system $A$}

The solution of aldehyde $1(0.29 \mathrm{~g}, 2.0 \mathrm{mmol})$ and ketone $\mathbf{3 f}, \mathbf{g}(2.0 \mathrm{mmol})$ in benzene $(4 \mathrm{~mL})$ was added dropwise to the stirred mixture of $50 \%$ aq $\mathrm{NaOH}(1 \mathrm{~mL}, 19.1 \mathrm{mmol})$, TEBAC $(0.023 \mathrm{~g}$, $0.1 \mathrm{mmol})$ and benzene $(6 \mathrm{~mL})$. When aldehyde 1 was no longer detected by $\mathrm{GC}(1 \mathrm{~h})$, the reaction mixture was diluted with water $(50 \mathrm{~mL})$. The organic phase was separated and the water phase was extracted with $\mathrm{CHCl}_{3}(3 \times 20 \mathrm{~mL})$. Combined organic phases were washed with water $(30 \mathrm{~mL})$ and dried $\left(\mathrm{MgSO}_{4}\right)$. After the solvent was evaporated, the crude dark mixture was purified by column chromatography and naphthalenes 6f,g (characterized below) were obtained, however in a much lower yield (Table 1) than in system B.

\section{General procedure for preparation of 1-cyano-3-acylnaphthalenes 6f,g in system B}

To the stirred solution of aldehyde $1(0.29 \mathrm{~g}, 2.0 \mathrm{mmol})$, vinyl ketone $\mathbf{3 f}, \mathbf{g}(2.0 \mathrm{mmol})$ and Aliquat $336(0.040 \mathrm{~g}, 0.1 \mathrm{mmol})$ in benzene $(30 \mathrm{~mL}), \mathrm{K}_{2} \mathrm{CO}_{3}(0.138 \mathrm{~g}, 10.0 \mathrm{mmol})$ was added. When aldehyde 1 was no longer detected by GC (ca $2 \mathrm{~h}$ ), the reaction mixture has been worked up as described in General procedure for 6a-e in system A. The crude mixture was purified by column chromatography (Table 1) and the products were recrystallized to give analytical samples of $\mathbf{6 f}, \mathbf{g}$.

1-Cyano-3-acetylnaphthalene (6f). M.p. $156-157^{\circ} \mathrm{C}$ (benzene/cyclohexane). Yield: $281 \mathrm{mg}(72$ \%). ${ }^{1} \mathrm{H}$ NMR: $\delta=2.74\left(\mathrm{~s}, 3 \mathrm{H}, \mathrm{CH}_{3}\right), 7.68-7.87\left(\mathrm{~m}, 2 \mathrm{H}, \mathrm{CH}_{\mathrm{Ar}}\right), 8.08\left(\mathrm{~d}, J=8.0,1 \mathrm{H}, \mathrm{CH}_{\mathrm{Ar}}\right), 8.28$ $\left(\mathrm{d}, J=8.0,1 \mathrm{H}, \mathrm{CH}_{\mathrm{Ar}}\right), 8.47\left(\mathrm{~d}, J=1.6,1 \mathrm{H}, \mathrm{CH}_{\mathrm{Ar}}\right), 8.66\left(\mathrm{~s}, 1 \mathrm{H}, \mathrm{CH}_{\mathrm{Ar}}\right) \mathrm{ppm} .{ }^{13} \mathrm{C} \mathrm{NMR:} \delta=$ 26.5, 111.1, 117.02, 125.2, 128.5, 130.3, 130.9, 131.1, 132.2, 133.6, 133.9, 134.4, 195.8 ppm. IR: $v=3072,2220,1680,1620,1292,900,752 \mathrm{~cm}^{-1} . \mathrm{C}_{13} \mathrm{H}_{9} \mathrm{NO}$ (195.2): calcd. C 79.98, H 4.65, N 7.17; found: C 79.89, H 4.71, N 7.05.

1-Cyano-3-propionylnaphthalene (6g). Mp 104-105 ${ }^{\circ} \mathrm{C}$ (AcOEt). Yield: $289 \mathrm{mg}(60 \%) .{ }^{1} \mathrm{H}$ NMR: $\delta=1.30\left(\mathrm{t}, J=7.2,3 \mathrm{H}, \mathrm{CH}_{3}\right), 3.14\left(\mathrm{q}, J=7.2,2 \mathrm{H}, \mathrm{CH}_{2}\right), 7.66-7.85\left(\mathrm{~m}, 3 \mathrm{H}, \mathrm{CH}_{\mathrm{Ar}}\right), 8.06$ $\left(\mathrm{d}, J=7.6,1 \mathrm{H}, \mathrm{CH}_{\mathrm{Ar}}\right), 8.26\left(\mathrm{~d}, J=7.6,1 \mathrm{H}, \mathrm{CH}_{\mathrm{Ar}}\right), 8.45\left(\mathrm{~d}, J=1.6,1 \mathrm{H}, \mathrm{CH}_{\mathrm{Ar}}\right), 8.67$ (br. s, $1 \mathrm{H}$, $\mathrm{CH}_{\mathrm{Ar}}$ ) ppm. ${ }^{13} \mathrm{C}$ NMR: $\delta=8.09,31.9,111.1,117.1,125.2,128.4,130.3,130.89,130.90,132.3$, 133.4, 133.8, 133.9, 198.5 ppm. IR: $v=3064,2980,2220,1696,1620,1184,748 \mathrm{~cm}^{-1}$. $\mathrm{C}_{14} \mathrm{H}_{11} \mathrm{NO}$ (209.2): calcd. C 80.36, H 5.30, N 6.69; found: C 80.23, H 5.55, N 6.66.

\section{General procedure for preparation of 1-substituted-3-cyanonaphthalenes $10 \mathrm{~h}, \mathrm{i}$}

To the stirred solution of aldehyde $1(0.29 \mathrm{~g}, 2.0 \mathrm{mmol})$, acrylonitrile $(3 \mathrm{~h}, 0.11 \mathrm{~g}, 2.0 \mathrm{mmol})$ or phenyl vinyl sulfone (3i, $0.739 \mathrm{~g}, 4.4 \mathrm{mmol})$ and TEBAC $(0.023 \mathrm{~g}, 0.1 \mathrm{mmol})$ in benzene $(30$ $\mathrm{mL}), 50 \%$ aq $\mathrm{NaOH}(1 \mathrm{~mL}, 19.1 \mathrm{mmol})$ was added. When aldehyde 1 was no longer detected by GC $(2 \mathrm{~h})$, the reaction mixture has been worked up as described for compounds $\mathbf{6} \mathbf{f}, \mathbf{g}$ and the 
crude mixture was purified by column chromatography (Table 1). The products were recrystallized to give analytical samples of $\mathbf{1 0 h , i}$.

1-(2-Cyanoethyl)-3-cyanonaphthalene (10h). Mp 93-94 ${ }^{\circ} \mathrm{C}$ (AcOEt). Yield: $140 \mathrm{mg}(34 \%) .{ }^{1} \mathrm{H}$ NMR: $\delta=2.80\left(\mathrm{t}, J=12.6,2 \mathrm{H}, \mathrm{CH}_{2}\right), 3.46\left(\mathrm{t}, J=12.6,2 \mathrm{H}, \mathrm{CH}_{2}\right), 7.53\left(\mathrm{~d}, J=0.8,1 \mathrm{H}, \mathrm{CH}_{\mathrm{Ar}}\right)$, 7.63-7.68 (m, $\left.1 \mathrm{H}, \mathrm{CH}_{\mathrm{Ar}}\right), 7.72-7.76\left(\mathrm{~m}, 1 \mathrm{H}, \mathrm{CH}_{\mathrm{Ar}}\right), 7.95\left(\mathrm{~d}, J=5.2,1 \mathrm{H}, \mathrm{CH}_{\mathrm{Ar}}\right), 8.37(\mathrm{~d}, J=$ 5.2, $1 \mathrm{H}, \mathrm{CH}_{\mathrm{Ar}}$ ), 8.19 (br. s, $1 \mathrm{H}, \mathrm{CH}_{\mathrm{Ar}}$ ) ppm. ${ }^{13} \mathrm{C} \mathrm{NMR}: \delta=18.1,28.3,109.1,118.4,118.8$, $122.9,126.5,127.6,129.7,132.5,132.7,134.1,135.8$ ppm. IR: $v=3064,2948,2248,2220$, 1600, 1224, 884, $752 \mathrm{~cm}^{-1} . \mathrm{C}_{14} \mathrm{H}_{10} \mathrm{~N}_{2}$ (206.2): calcd. C 81.53, H 4.89, N 13.58; found: C 81.16, H 5.21, N 13.33 .

1-(2-Phenylsulfonylethyl)-3-(phenylsulfonyl)naphthalene (10i). Mp 185-186 ${ }^{\circ} \mathrm{C}$ (acetone). Yield: $680 \mathrm{mg}(78 \%)$; ${ }^{1} \mathrm{H}$ NMR: $\delta=3.41-3.46\left(\mathrm{~m}, 2 \mathrm{H}, \mathrm{CH}_{2}\right), 3.50-3.55\left(\mathrm{~m}, 2 \mathrm{H}, \mathrm{CH}_{2}\right)$, 7.45$7.72\left(\mathrm{~m}, 10 \mathrm{H}, \mathrm{CH}_{\mathrm{Ar}}\right), 7.87-8.01\left(\mathrm{~m}, 5 \mathrm{H}, \mathrm{CH}_{\mathrm{Ar}}\right), 8.44\left(\mathrm{~d}, J=1.6,1 \mathrm{H}, \mathrm{CH}_{\mathrm{Ar}}\right) \mathrm{ppm} .{ }^{13} \mathrm{C} \mathrm{NMR:} \delta=$ 26.0, 56.0, 122.7, 123.0, 127.6, 128.0, 128.9, 129.3, 129.4, 130.6, 132.7, 132.9, 133.3, 134.0, 135.9, 138.0, 138.7, 141.2 ppm. IR: $v=3060,2968,1584,1444,1304,1132,752 \mathrm{~cm}^{-1}$. $\mathrm{C}_{24} \mathrm{H}_{20} \mathrm{O}_{4} \mathrm{~S}_{2}$ (436.6): calcd. C 66.02, H 4.63, S 14.69; found: C 65.85, H 4.41, S 14.59.

Preparation of 1-cyano-10-methyltetracyclo[7.5.1.0 $\left.{ }^{2,7} \cdot 0^{\mathbf{8}, 13}\right]$ pentadeca-2,4,6,10-tetraen-12one (11). To the stirred solution of aldehyde 1 ( $0.29 \mathrm{~g}, 2.0 \mathrm{mmol})$, methyl vinyl ketone (3f, 0.42 $\mathrm{g}, 6.0 \mathrm{mmol})$ and TEBAC $(0.023 \mathrm{~g}, 0.1 \mathrm{mmol})$ in benzene $(30 \mathrm{~mL}), 50 \%$ aq $\mathrm{NaOH}(1 \mathrm{~mL}, 19.1$ mmol) was added. The stirring was continued at $\mathrm{rt}$ for $3 \mathrm{~h}$. The reaction mixture was diluted with water $(50 \mathrm{~mL})$ and has been worked up as described for naphthalenes $6 \mathbf{6}, \mathbf{g}$. After the solvent was evaporated, the crude mixture was purified by column chromatography. The product was recrystallized to give analytical sample of 11. Mp 109-110 ${ }^{\circ} \mathrm{C}$ (cyclohexane). Yield: $448 \mathrm{mg}$ (90 \%). ${ }^{1} \mathrm{H}$ NMR: $\delta=1.95-2.18\left(\mathrm{~m}, 4 \mathrm{H}, 2 \times \mathrm{CH}_{2}\right), 2.05\left(\mathrm{~d}, J=1.0,3 \mathrm{H}, \mathrm{CH}_{3}\right), 2.28-2.38(\mathrm{~m}, 2 \mathrm{H}$, $2 \times \mathrm{CH}), 3.55(\mathrm{t}, J=3.0,1 \mathrm{H}, \mathrm{CH}), 5.80(\mathrm{br} . \mathrm{s}, 1 \mathrm{H},=\mathrm{CH}), 7.27-7.30\left(\mathrm{~m}, 1 \mathrm{H}, \mathrm{CH}_{\mathrm{Ar}}\right), 7.35-7.42(\mathrm{~m}$, $\left.2 \mathrm{H}, \mathrm{CH}_{\mathrm{Ar}}\right), 7.60\left(\mathrm{dd}, J=7.0, J=1.0,1 \mathrm{H}, \mathrm{CH}_{\mathrm{Ar}}\right) \mathrm{ppm} .{ }^{13} \mathrm{C} \mathrm{NMR}: \delta=23.5,32.5,35.3,36.6$, 37.0, 39.9, 41.5, 120.3, 121.4, 122.2, 124.9, 127.8, 128.3, 136.7, 138.4, 166.8, 200.1 ppm. IR: $v$ $=3032,2940,2240,1672,1436,1380,880,760 \mathrm{~cm}^{-1} . \mathrm{C}_{17} \mathrm{H}_{15} \mathrm{NO}$ (249.3): calcd. C 81.90, H 6.06, N 5.62; found: C 81.84, H 6.09, N 5.59.

\section{General procedure for preparation of cyanoketones 13a,b}

These compounds were obtained according to General procedure in system B, starting from aldehyde 1 (0.29 g, $2.0 \mathrm{mmol})$, cyclic ketone 12a,b (8.0 mmol), Aliquat 336 (0.040 g, $0.1 \mathrm{mmol})$, benzene $(30 \mathrm{~mL})$ and powdered $\mathrm{K}_{2} \mathrm{CO}_{3}(0.138 \mathrm{~g}, 10.0 \mathrm{mmol})$. The crude mixture was purified by column chromatography and the products were recrystallized to give analytical samples of 13a,b. 4-Cyano-2,3-dihydro-cyclopenta[b]naphthalene-1-one (13a). Mp $207^{\circ} \mathrm{C}$ (decomp.) (AcOEt). Yield: $228 \mathrm{mg}(55 \%) .{ }^{1} \mathrm{H}$ NMR: $\delta=2,88-2,92\left(\mathrm{~m}, 2 \mathrm{H}, \mathrm{CH}_{2}\right), 3,51-3,54\left(\mathrm{~m}, 2 \mathrm{H}, \mathrm{CH}_{2}\right)$, 7.64$7.68\left(\mathrm{~m}, 1 \mathrm{H}, \mathrm{CH}_{\mathrm{Ar}}\right), 7.81-7.85\left(\mathrm{~m}, 1 \mathrm{H}, \mathrm{CH}_{\mathrm{Ar}}\right), 8.08\left(\mathrm{~d}, J=8.4,1 \mathrm{H}, \mathrm{CH}_{\mathrm{Ar}}\right), 8.27(\mathrm{~d}, J=8.4,1 \mathrm{H}$, $\left.\mathrm{CH}_{\mathrm{Ar}}\right), 8.50\left(\mathrm{~s}, 1 \mathrm{H}, \mathrm{CH}_{\mathrm{Ar}}\right), 8.50\left(\mathrm{~s}, 1 \mathrm{H}, \mathrm{CH}_{\mathrm{Ar}}\right) \mathrm{ppm} .{ }^{13} \mathrm{C} \mathrm{NMR}: \delta=25.4,36.3,107.6,115.5$, $125.0,127.7,129.3,131.3,131.4,132.0,134.4,135.8,154.5,204.7$ ppm. IR: $v=3060,2924$, 
2220, 1712, 1620, 1172, $764 \mathrm{~cm}^{-1} . \mathrm{C}_{14} \mathrm{H}_{9} \mathrm{NO}$ (207.2): calcd. C 81.14, H 4.38, N 6.76; found: $\mathrm{C}$ $80.59, \mathrm{H} 4.50, \mathrm{~N} 6.63$.

10-Cyano-3,4-dihydro-2H-anthracen-1-one (13b). $\mathrm{Mp} \mathrm{143-145}{ }^{\circ} \mathrm{C}$ (AcOEt). Yield: $354 \mathrm{mg}$ (80 \%). ${ }^{1} \mathrm{H}$ NMR $(200 \mathrm{MHz}): \delta=2.15-2.34\left(\mathrm{~m}, 2 \mathrm{H}, \mathrm{CH}_{2}\right), 2.77-2.83\left(\mathrm{~m}, 2 \mathrm{H}, \mathrm{CH}_{2}\right), 3.37-3.43$ $\left(\mathrm{m}, 2 \mathrm{H}, \mathrm{CH}_{2}\right), 7.58-7.7 .66\left(\mathrm{~m}, 1 \mathrm{H}, \mathrm{CH}_{\mathrm{Ar}}\right), 7.74-7.83\left(\mathrm{~m}, 1 \mathrm{H}, \mathrm{CH}_{\mathrm{Ar}}\right), 8.04(\mathrm{~d}, J=8.2,1 \mathrm{H}$, $\left.\mathrm{CH}_{\mathrm{Ar}}\right), 8.22\left(\mathrm{~d}, J=8.2,1 \mathrm{H}, \mathrm{CH}_{\mathrm{Ar}}\right), 8.81\left(\mathrm{~s}, 1 \mathrm{H}, \mathrm{CH}_{\mathrm{Ar}}\right) \mathrm{ppm} .{ }^{13} \mathrm{C} \mathrm{NMR}: \delta=22.3,29.0,38.9$, 109.2, 124.9, 127.6, 128.3, 130.2, 130.8, 131.1, 131.3, 133.6, 134.9, 146.0, 196.5 ppm. IR: $v=$ 3056, 2948, 2216, 1688, 1620, 1176, $748 \mathrm{~cm}^{-1}$. $\mathrm{C}_{15} \mathrm{H}_{11} \mathrm{NO}$ (221.3): calcd. C 81.43, H 5.01, N 6.33; found: C 81.28, H 5.08, N 6.40 .

\section{General procedure for preparation of 1-cyano-5,8-diaroylbenzobicyclo[2.2.2]}

The reactions were carried out as described for $\mathbf{6}$ in General procedure in system A, starting from aldehyde 1 ( $0.29 \mathrm{~g}, 2.0 \mathrm{mmol})$, chloroketone $2 \mathrm{a}-\mathrm{e}(4.2 \mathrm{mmol})$, TEBAC $(0.023 \mathrm{~g}, 0.1 \mathrm{mmol})$, benzene $(30 \mathrm{~mL})$ and $50 \%$ aq $\mathrm{NaOH}(1 \mathrm{~mL}, 19.1 \mathrm{mmol})$. The crude mixtures were purified by column chromatography and the products $\mathbf{9 a - e}$ were recrystallized. In case of the reactions of $\mathbf{2 a}$ and 2e, minute amounts (ca. 5\%) of the corresponding naphthalenes 3a and 3e were isolated (Table 1).

1-Cyano-5,8-dibenzoyl-benzobicyclo[2.2.2]octene (9a). $\mathrm{Mp} 165{ }^{\circ} \mathrm{C}$ (AcOEt). Yield: $470 \mathrm{mg}$ (60 \%). ${ }^{1} \mathrm{H}$ NMR: $\delta=2.08-2.14\left(\mathrm{~m}, 1 \mathrm{H}, \mathrm{CH}_{2}\right), 2.36-2.48\left(\mathrm{~m}, 2 \mathrm{H}, \mathrm{CH}_{2}\right), 2.82$ (dd, $J=12.8, J=$ 6.4, $1 \mathrm{H}, \mathrm{CH}_{2}$ ), 3.59 (ddd, $\left.J=11.2, J=6.4, J=2.4,1 \mathrm{H}, \mathrm{CH}\right), 3.73(\mathrm{~m}, 1 \mathrm{H}, \mathrm{CH}), 4.14$ (ddd, $J=$ 9.6, $J=5.6, J=2.0,1 \mathrm{H}, \mathrm{CH}), 7.00\left(\mathrm{~d}, J=7.2,1 \mathrm{H}, \mathrm{CH}_{\mathrm{Ar}}\right), 7.26-7.42\left(\mathrm{~m}, 5 \mathrm{H}, \mathrm{CH}_{\mathrm{Ar}}\right), 7.49-7.70$ $\left(\mathrm{m}, 4 \mathrm{H}, \mathrm{CH}_{\mathrm{Ar}}\right), 7.73\left(\mathrm{~d}, J=8.0,2 \mathrm{H}, \mathrm{CH}_{\mathrm{Ar}}\right), 8.00\left(\mathrm{~d}, J=8.0,2 \mathrm{H}, \mathrm{CH}_{\mathrm{Ar}}\right)$ ppm. ${ }^{13} \mathrm{C}$ NMR: $\delta=$ $32.6,32.7,36.5,39.4,40.0,44.4,120.7,122.3,125.0,127.96,128.0,128.18,128.23,128.3$, 129.0, 133.0, 133.8, 135.2, 135.6, 136.4, 136.6, 198.0, 199.6 ppm. IR: $v=3060,2948,2240$, 1672, 1448, 1220, 1016, $756 \mathrm{~cm}^{-1}$. $\mathrm{C}_{27} \mathrm{H}_{21} \mathrm{NO}_{2}$ (391.5): calcd. C 82.84, H 5.41, N 3.58; found: $\mathrm{C}$ 82.93, H 5.45, N 3.57 .

1-Cyano-5,8-di-(4-methylbenzoyl)-benzobicyclo[2.2.2] octene (9b). Mp 223-225 ${ }^{\circ} \mathrm{C}$ (AcOEt). Yield: $436 \mathrm{mg}(52 \%) .{ }^{1} \mathrm{H}$ NMR: $\delta=2.37-2.52\left(\mathrm{~m}, 4 \mathrm{H}, 2 \times \mathrm{CH}_{2}\right), 2.42\left(\mathrm{~s}, 6 \mathrm{H}, 2 \times \mathrm{CH}_{3}\right), 3.80(\mathrm{~s}, 1$ $\mathrm{H}, \mathrm{CH}), 4.04-4.07(\mathrm{~m}, 2 \mathrm{H}, \mathrm{CH}), 6.76\left(\mathrm{~d}, J=7.4,1 \mathrm{H}, \mathrm{CH}_{\mathrm{Ar}}\right), 7.15-7.19\left(\mathrm{~m}, 1 \mathrm{H}, \mathrm{CH}_{\mathrm{Ar}}\right), 7.27(\mathrm{~d}$, $\left.J=7.4,4 \mathrm{H}, \mathrm{CH}_{\mathrm{Ar}}\right), 7.30-7.34\left(\mathrm{~m}, 1 \mathrm{H}, \mathrm{CH}_{\mathrm{Ar}}\right), 7.53\left(\mathrm{~d}, J=8.0,1 \mathrm{H}, \mathrm{CH}_{\mathrm{Ar}}\right), 7.73(\mathrm{~d}, J=8.0,4 \mathrm{H}$, $\mathrm{CH}_{\mathrm{Ar}}$ ) ppm. ${ }^{13} \mathrm{C}$ NMR: $\delta=21.6,33.3,36.2,40.4,44.6,120.8,121.7,126.7,127.8,127.9,128.3$, 129.5, 133.0, 133.6, 144.2, 197.1 ppm. IR: $v=2944,2240,1664,1604,1236,984,768 \mathrm{~cm}^{-1}$. $\mathrm{C}_{29} \mathrm{H}_{25} \mathrm{NO}_{2}$ (419.5): calcd. C 83.03, H 6.01, N 3.34; found: C 83.13, H 6.18, N 3.22.

1-Cyano-5,8-di-(4-chlorobenzoyl)-benzobicyclo[2.2.2] $]$ ctene (9c). Mp 203-206 ${ }^{\circ} \mathrm{C}$ (AcOEt). Yield: $718 \mathrm{mg}\left(78 \%\right.$ ). ${ }^{1} \mathrm{H}$ NMR (Varian Gemini 200BB, $200 \mathrm{MHz}, \mathrm{CDCl}_{3}$ ): $\delta=2.11-2.30(\mathrm{~m}, 1$ $\mathrm{H}, \mathrm{CH}_{2}$ ), 2.36-2.45 (m, $2 \mathrm{H}, \mathrm{CH}_{2}$ ), 2.66 (dd, $J=12.7, J=6.5,1 \mathrm{H}, \mathrm{CH}_{2}$ ), 3.50 (ddd, $J=11.2, J=$ $6.4, J=2.1,1 \mathrm{H}, \mathrm{CH}), 3.65(\mathrm{~m}, 1 \mathrm{H}, \mathrm{CH}), 4.17$ (ddd, $J=9.0, J=6.5, J=2.0,1 \mathrm{H}, \mathrm{CH}), 6.99(\mathrm{~d}$, $\left.J=7.2,1 \mathrm{H}, \mathrm{CH}_{\mathrm{Ar}}\right), 7.25-7.44\left(\mathrm{~m}, 4 \mathrm{H}, \mathrm{CH}_{\mathrm{Ar}}\right), 7.52\left(\mathrm{~d}, J=8.6,2 \mathrm{H}, \mathrm{CH}_{\mathrm{Ar}}\right), 7.61(\mathrm{~d}, J=7.2,1 \mathrm{H}$, $\left.\mathrm{CH}_{\mathrm{Ar}}\right), 7.77\left(\mathrm{~d}, J=8.6,2 \mathrm{H}, \mathrm{CH}_{\mathrm{Ar}}\right), 7.90\left(\mathrm{~d}, J=8.6, \mathrm{CH}_{\mathrm{Ar}}\right) \mathrm{ppm} .{ }^{13} \mathrm{C} \mathrm{NMR}: \delta=33.1,36.6,39.4$, $39.7,44.5,120.5,122.6,125.0,128.2,128.3,129.0,129.5,129.78,129.83,133.6,133.9,136.2$, 
136.5, 139.7, 140.5, 197.0, 198.7 ppm. IR: $v=3070,2956,2240,1680,1588,1400,1224,1092$, $764 \mathrm{~cm}^{-1} . \mathrm{C}_{27} \mathrm{H}_{19} \mathrm{NO}_{2} \mathrm{Cl}_{2}$ (460.4): calcd. C 70.44, H 4.16, N 3.04, Cl 15.40; found: C 70.25, $\mathrm{H}$ 4.34, N 2.85, Cl 15.20.

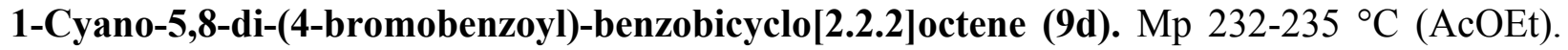
Yield: $549 \mathrm{mg}(50 \%) .{ }^{1} \mathrm{H}$ NMR: $\delta=2.14-2.21\left(\mathrm{~m}, 1 \mathrm{H}, \mathrm{CH}_{2}\right), 2.33-2.44\left(\mathrm{~m}, 2 \mathrm{H}, \mathrm{CH}_{2}\right), 2.65$ (dd, $\left.J=13.0, J=6.6,1 \mathrm{H}, \mathrm{CH}_{2}\right), 3.49(\mathrm{ddd}, J=11.5, J=6.6, J=2.4,1 \mathrm{H}, \mathrm{CH}), 3.65(\mathrm{~m}, 1 \mathrm{H}, \mathrm{CH})$, $4.15(\mathrm{ddd}, J=9.8, J=5.8, J=1.6,1 \mathrm{H}, \mathrm{CH}), 6.99\left(\mathrm{~d}, J=7.4,1 \mathrm{H}, \mathrm{CH}_{\mathrm{Ar}}\right), 7.29(\mathrm{dd}, J=7.4, J=$ 7.6, $\left.1 \mathrm{H}, \mathrm{CH}_{\mathrm{Ar}}\right), 7.40\left(\mathrm{dd}, J=7.4, J=7.6,1 \mathrm{H}, \mathrm{CH}_{\mathrm{Ar}}\right), 7.53\left(\mathrm{~d}, J=8.4,2 \mathrm{H}, \mathrm{CH}_{\mathrm{Ar}}\right), 7.61(\mathrm{~d}, J=$ 7.6, $\left.1 \mathrm{H}, \mathrm{CH}_{\mathrm{Ar}}\right), 7.69\left(\mathrm{~d}, J=8.4,4 \mathrm{H}, \mathrm{CH}_{\mathrm{Ar}}\right), 7.82\left(\mathrm{~d}, J=8.4,2 \mathrm{H}, \mathrm{CH}_{\mathrm{Ar}}\right) \mathrm{ppm} .{ }^{13} \mathrm{C} \mathrm{NMR:} \delta=$ 33.0, 33.1, 36.6, 39.4, 39.7, 44.4, 120.5, 122.6, 125.0, 128.3, 128.5, 129.3, 129.88, 129.94, 132.1, $132.5,134.0,134.2,136.2,136.4,197.2,198.9$ ppm. IR: $v=3072,2956,2240,1672,1584$, 1392, 1228, 1068, $760 \mathrm{~cm}^{-1} . \mathrm{C}_{21} \mathrm{H}_{19} \mathrm{BrNO}_{2}$ (397.3): calcd. C 59.04, H 3.49, N 2.55, Br 29.10; found: C 58.73, H 3.54, N 2.45, Br 29.18.

1-Cyano-5,8-di-(3,4-dimethoxybenzoyl)-benzobicyclo[2.2.2]octene (9e). Mp 134-138 ${ }^{\circ} \mathrm{C}$ (AcOEt). Yield: $481 \mathrm{mg}(47 \%) .{ }^{1} \mathrm{H}$ NMR: $\delta=2.09-2.16\left(\mathrm{~m}, 1 \mathrm{H}, \mathrm{CH}_{2}\right), 2.34-2.40(\mathrm{~m}, 2 \mathrm{H}$, $\left.\mathrm{CH}_{2}\right), 2.71\left(\mathrm{dd}, J=12.8, J=6.4,1 \mathrm{H}, \mathrm{CH}_{2}\right), 3.50-3.55(\mathrm{~m}, 1 \mathrm{H}, \mathrm{CH}), 3.71(\mathrm{~s}, 1 \mathrm{H}, \mathrm{CH}), 3.78(\mathrm{~s}, 3$ $\left.\mathrm{H}, \mathrm{OCH}_{3}\right), 3.90\left(\mathrm{~s}, 3 \mathrm{H}, \mathrm{OCH}_{3}\right), 3.96\left(\mathrm{~s}, 3 \mathrm{H}, \mathrm{OCH}_{3}\right), 3.97\left(\mathrm{~s}, 3 \mathrm{H}, \mathrm{OCH}_{3}\right), 4.18-4.23(\mathrm{~m}, 1 \mathrm{H}$, $\mathrm{CH}), 6.78\left(\mathrm{~d}, J=8.4,1 \mathrm{H}, \mathrm{CH}_{\mathrm{Ar}}\right), 6.91\left(\mathrm{~d}, J=8.4,1 \mathrm{H}, \mathrm{CH}_{\mathrm{Ar}}\right), 7.03\left(\mathrm{~d}, J=7.2,1 \mathrm{H}, \mathrm{CH}_{\mathrm{Ar}}\right), 7.29$ $\left(\mathrm{d}, J=7.2,1 \mathrm{H}, \mathrm{CH}_{\mathrm{Ar}}\right), 7.36-7.40\left(\mathrm{~m}, 2 \mathrm{H}, \mathrm{CH}_{\mathrm{Ar}}\right), 7.49-7.53\left(\mathrm{~m}, 2 \mathrm{H}, \mathrm{CH}_{\mathrm{Ar}}\right), 7.59-7.60(\mathrm{~m}, 2 \mathrm{H}$, $\mathrm{CH}_{\mathrm{Ar}}$ ) ppm. ${ }^{13} \mathrm{C} \mathrm{NMR:} \delta=33.2,33.4,36.6,38.9,40.7,44.1,55.8,56.0,56.1,56.2,110.0,110.3$, $110.4,110.7,120.8,122.4,122.9$, 123.0, 125.0, 127.9, 128.0, 128.4, 128.7, 136.4, 137.1, 148.9, 149.4, 153.2, 153.8, 196.8, 198.1 ppm. IR: $v=3084,2996,2224,1640,1512,1264,1024,772$ $\mathrm{cm}^{-1} . \mathrm{C}_{31} \mathrm{H}_{29} \mathrm{NO}_{6}(511.6)$ : calcd. C 72.78, H 5.71, N 2.74; found: C 72.89, H 5.88, N 2.70.

Preparation of 1-cyano-3-benzoyl-1,2-dihydronaphthalene (5a) and its dehydrogenation into 1-cyano-3-benzoylnaphthalene (6a)

[2-(2,5-Dioxacyclopentyl)-phenyl]-acetonitrile (14). The mixture of aldehyde 1 (3.05 g, 21.0 $\mathrm{mmol})$, ethylene glycol (1.93 g, $31.5 \mathrm{mmol})$, p-toluenesulfonic acid $(0.21 \mathrm{~g}, 1.1 \mathrm{mmol})$ in benzene $(50 \mathrm{~mL})$ was refluxed for $6 \mathrm{~h}$ and water was removed by Dean-Stark trap. Then the reaction mixture was cooled, washed with saturated aq. solution of $\mathrm{NaHCO}_{3}(2 \times 30 \mathrm{~mL})$, water $(2 \times 20 \mathrm{~mL})$, dried $\left(\mathrm{MgSO}_{4}\right)$ and the solvent was evaporated to give product 14 as pale yellow, sticky oil (yield $3.81 \mathrm{~g}, 96 \%$ ). ${ }^{1} \mathrm{H}$ NMR: $\delta=3.96\left(\mathrm{~s}, 2 \mathrm{H}, \mathrm{CH}_{2} \mathrm{CN}\right), 4.02-4.16\left(\mathrm{~m}, 4 \mathrm{H}, \mathrm{CH}_{2} \mathrm{CH}_{2}\right.$ ), $5.85(\mathrm{~s}, 1 \mathrm{H}, \mathrm{OCHO}), 7.30-7.58\left(\mathrm{~m}, 4 \mathrm{H}, \mathrm{CH}_{\mathrm{Ar}}\right) \mathrm{ppm} .{ }^{13} \mathrm{C} \mathrm{NMR}: \delta=20.7,64.9,102.5,117.8$, 127.6, 127.8, 128.7, 129.1, 129.6, 134.5 ppm. IR: $v=2956,2892,2248,1404,1224,1080,760$ $\mathrm{cm}^{-1} . \mathrm{C}_{11} \mathrm{H}_{11} \mathrm{NO}_{2}$ (189.2): calcd. C 69.83, H 5.86, N 7.40; found: C 69.84, H 5.85, N 7.43.

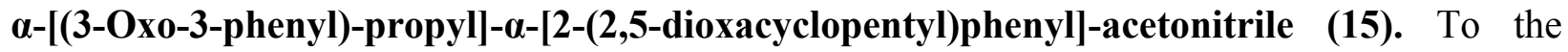
stirred solution of nitrile $\mathbf{1 4}(0.38 \mathrm{~g}, 2.0 \mathrm{mmol})$, ketone $\mathbf{2 a}(0.34 \mathrm{~g}, 2.0 \mathrm{mmol})$ and TEBAC $(0.023$ $\mathrm{g}, 0.1 \mathrm{mmol})$ in benzene $(30 \mathrm{~mL}), 50 \%$ aq $\mathrm{NaOH}(1 \mathrm{~mL}, 19.1 \mathrm{mmol})$ was added. The reaction was carried out for $2.0 \mathrm{~h}$, worked up as described for $\mathbf{6}$ in General procedure in system A, and the crude mixture was purified by column chromatography. The product 15 was obtained as 
transparent, colourless oil (yield $0.22 \mathrm{~g}, 35 \%$ ), which after being treated with EtOH, afforded crystals (m.p. $\left.58-61{ }^{\circ} \mathrm{C} ; 17 \%\right) .{ }^{1} \mathrm{H}$ NMR: $\delta=2.28-2.46\left(\mathrm{~m}, 2 \mathrm{H}, \mathrm{CH}_{2}\right), 3.20(\mathrm{t}, J=6.4 ; 2 \mathrm{H}$, $\left.\mathrm{COCH}_{2}\right), 3.97-4.15\left(\mathrm{~m}, 4 \mathrm{H}, \mathrm{OCH}_{2} \mathrm{CH}_{2} \mathrm{O}\right), 4.59$ (dd, $\left.J=9.2, J=6.4,1 \mathrm{H}, \mathrm{CHCN}\right), 5.94(\mathrm{~s}, 1 \mathrm{H}$, $\left.\mathrm{CHO}_{2}\right)$, 7.34-7.38 -7.61 (m, $\left.9 \mathrm{H}, \mathrm{CH}_{\mathrm{Ar}}\right), 7.94\left(\mathrm{~d}, J=1.2,1 \mathrm{H}, \mathrm{CH}_{\mathrm{Ar}}\right), 7.96\left(\mathrm{~s}, 1 \mathrm{H}, \mathrm{CH}_{\mathrm{Ar}}\right) \mathrm{ppm}$. ${ }^{13} \mathrm{C}$ NMR: $\delta=30.0,32.1,35.4,65.2,102.1,121.3,127.8,128.0,128.3,128.4,128.7,130.1$, 133.4, 134.6, 134.8, 136.6, 198.3 ppm. IR: $v=3060,2224,1644,1596,1444,1300,716 \mathrm{~cm}^{-1}$. $\mathrm{C}_{20} \mathrm{H}_{19} \mathrm{NO}_{3}$ (321.4): calcd. C 74.75, H 5.96, N 4.36; found: C 74.50, H 6.28, N 4.37.

1-Cyano-3-benzoyl-1,2-dihydronaphthalene (5a). The suspension of nitrile $\mathbf{1 5}(0.41 \mathrm{~g}, 1.32$ $\mathrm{mmol})$ in a mixture of acetonitrile $(6 \mathrm{~mL})$ and $3 \% \mathrm{HCl}(16 \mathrm{~mL})$ was refluxed for $2 \mathrm{~h}$, then cooled and diluted with water $(30 \mathrm{~mL})$. The mixture was extracted with $\mathrm{CH}_{2} \mathrm{Cl}_{2}(3 \times 20 \mathrm{~mL})$, the combined organic phases were washed with water $(2 \times 20 \mathrm{~mL})$ and dried $\left(\mathrm{MgSO}_{4}\right)$. The solvents were evaporated and the crude mixture was purified by column chromatography to give the product 5a as transparent, colourless oil (yield $0.27 \mathrm{~g}, 78 \%$ ). ${ }^{1} \mathrm{H}$ NMR: $\delta=3.02$ (part A of $\left.\mathrm{ABMX}, J_{\mathrm{AB}}=17.0, J_{\mathrm{AM}}=10.4, J_{\mathrm{AX}}=1.6,1 \mathrm{H}, \mathrm{CH}_{2}\right), 3.18\left(\right.$ part B of ABMX, $J_{\mathrm{AB}}=17.0, J_{\mathrm{BM}}=$ $7.2, J_{\mathrm{BX}}=0.8,1 \mathrm{H}, \mathrm{CH}_{2}$ ), $4.18\left(\right.$ part M of ABMX, $J_{\mathrm{AM}}=10.4, J_{\mathrm{BM}}=7.2,1 \mathrm{H}, \mathrm{CHCN}$ ), 7.23 (part $\mathrm{X}$ of $\mathrm{ABMX}$, br.s, $1 \mathrm{H},=\mathrm{CH}), 7.25\left(\mathrm{dd}, J_{\mathrm{H}, \mathrm{H}}=7.2,1.0,1 \mathrm{H}, \mathrm{CH}_{\mathrm{Ar}}\right), 7.35-7.40\left(\mathrm{~m}, 1 \mathrm{H}, \mathrm{CH}_{\mathrm{Ar}}\right)$, 7.41-7.46 (m, $\left.1 \mathrm{H}, \mathrm{CH}_{\mathrm{Ar}}\right), 7.48-7.56\left(\mathrm{~m}, 3 \mathrm{H}, \mathrm{CH}_{\mathrm{Ar}}\right), 7.57-7.62\left(\mathrm{~m} 2 \mathrm{H}, \mathrm{CH}_{\mathrm{Ar}}\right), 7.75-7.77(\mathrm{~m}, 1 \mathrm{H}$, $\mathrm{CH}_{\text {Ar }}$ ) ppm. ${ }^{13} \mathrm{C}$ NMR: $\delta=27.0,30.2,119.6,127.2,128.6$ (two C), 129.3 (three C), 129.6, 130.0, 131.0, 131.5, 132.2, 134.4, 137.4, 139.4, 196.0 ppm. IR: $v=3058,2224,1644,1596,1444$, $1228,752,716 \mathrm{~cm}^{-1} . \mathrm{C}_{18} \mathrm{H}_{13} \mathrm{NO}$ (259.3): calcd. C 83.38, H 5.05, N 5.40; found: C 83.29, H 5.25, N 5.40 .

1-Cyano-3-benzoylnaphthalene (6a). To the stirred solution of dihydronaphthalene $\mathbf{5 a}(0.27 \mathrm{~g}$, $1.03 \mathrm{mmol})$ and TEBAC $(0.02 \mathrm{~g}, 0.05 \mathrm{mmol})$ in benzene $(15 \mathrm{~mL}), 50 \%$ aq $\mathrm{NaOH}(0.5 \mathrm{~mL}, 9.5$ mmol) was added. The reaction was carried out for $2.0 \mathrm{~h}$, the mixture was diluted with water (25 $\mathrm{mL})$, the organic phase was separated, and the water phase was extracted with $\mathrm{CHCl}_{3}(3 \times 15 \mathrm{~mL})$. The combined organic phases were washed with water $(20 \mathrm{~mL})$ and dried $\left(\mathrm{MgSO}_{4}\right)$. The solvent was evaporated, the residue was purified by column chromatography and the product 6a was obtained as transparent colourless oil, which solidified. Recrystallization from methanol afforded the product, which exhibited physical and spectral properties the same as described for 6a obtained according to the General procedure in system A, (yield $0.23 \mathrm{~g}, 85 \%$ ).

\section{References}

1. Jończyk, A.; Zdrojewski, T.; Panasiewicz, M. Synth. Commun. 2003, 33, 4095.

2. Zdrojewski, T.; Jończyk, A. Tetrahedron 1995, 45, 12439.

3. Dehmlow, E. V.; Dehmlow, S. S. Phase Transfer Catalysis, 3rd Ed., Verlag Chemie: Weinheim, 1993.

4. Starks, C. M.; Liotta, C. L.; Halpern, M. Phase-Transfer Catalysis, Chapman \& Hall: New York, 1994. 
5. Mąkosza, M.; Fedoryński, M. Cat. Rev. 2003, 45, 321; idem, ARKIVOC 2006, (iv), 7.

6. Jończyk, A. ARKIVOC 2004, (iii), 176.

7. Severin, T.; Schmitz, R.; Temme, H.-L. Chem. Ber. 1963, 96, 2499.

8. Squadrito, G. L.; Fronczek, F. R.; Church, D. F.; Pryou, W. A. J. Org. Chem. 1989, 54, 548.

9. Banciu, M. D.; Brown, R. F. C.; Coulston, K. J.; Eastwood, F. W.; Macrae, T. Aust. J. Chem. 1998, 51, 695.

10. Barker, S. D.; Wilson, K.; Norris, R. K. Aust. J. Chem. 1995, 48, 1969.

11. Dewar, M. J. S.; Grisdale, P. J. J. Am. Chem. Soc. 1962, 84, 3541.

12. Hori, M.; Kataoka, T.; Shimizu, H.; Narita, K.; Ohno, S.; Ogura, H.; Takayanagi, H.; Iitaka, Y.; Koyama, H. J. Chem. Soc., Perkin Trans. 1 1988, 1885.

13. Hren, J.; Polanc, S.; Kočevar, M. ARKIVOC 2008, (i), 209.

14. Masuyama, Y.; Ueno, Y.; Okawara, M. Chem. Lett. 1977, 1439.

15. Donetti, A.; Boniardi, O.; Ezhaya, A. Synthesis 1980, 1009.

16. Hermann, C. K. F.; Sachdeva, Y. P.; Wolfe, J. F. J. Heterocyclic Chem. 1987, 24, 1061.

17. Fasani, E.; d'Alessandro, N.; Albini, A.; Mariano, P. S. J. Org. Chem. 1994, 59, 829.

18. Govindachari, T. R.; Ragade, I.S.; Viswanathan, N. J. Chem. Soc. 1962, 1357.

19. Horner, M.; Hünig, S. Liebigs Ann. Chem. 1982, 1183.

20. Coulston, K. J.; Eastwood, F. W.; Jurss, C. Aust. J. Chem. 1996, 49, 965.

21. Bruson, H. A. Org. React. 1949, 5, 79.

22. Bergmann, E. D.; Ginsburg, D.; Rappo, R. Org. React. 1959, 10, 179.

23. Arseniyadis, S.; Kyler, K. S.; Watt, D. S. Org. React. 1984, 31, 1.

24. Kitahonoki, H.; Takano, Y. Tetrahedron Lett. 1963, 24, 1597.

25. Tabushi, I.; Yoshida, Z.; Aoyama, Y. Bull. Chem. Soc. Japan 1974, 74, 3079.

26. Butler, D. N.; Koves, G. Synth. Commun. 1975, 5, 471.

27. Gleiter, R.; Krennrich, G.; Bischof, P.; Tsuji, T.; Nishida, S. Helv. Chim. Acta 1986, 69, 962.

28. Tsuji, T.; Nishida, S. J. Am. Chem. Soc. 1988, 110, 2157.

29. Haddon, V. R.; Chen, H. Tetrahedron Lett. 1976, 51, 4669.

30. Gravel, D.; Giasson, R.; Blanchet, D.; Yip, R. W.; Sharma, D. K. Can. J. Chem. 1991, 69, 1193.

31. Peña-Cabrera, E.; Norrby, P.-O.; Sjögren, M.; Vitagliano, A.; De Felice, V.; Oslob, J.; Ishii, S.; O'Neill, D.; Åkermark, B.; Helquist, P. J. Am. Chem. Soc. 1996, 118, 4299.

32. Ito, Y.; Nakatsuka, M.; Saegusa, T. J. Am. Chem. Soc. 1982, 104, 7609.

33. Di Valentin, C.; Freccero, M.; Sarzi-Amadè, M.; Zanaletti R. Tetrahedron 2000, 56, 2547.

34. Johnson, R. P.; Exarchou, A.; Jefford, C. W.; Hahn R. C. J. Org. Chem. 1977, 42, 3758.

35. Ju, J.-W.; Huang S. K. Org. Prep. Proc. Int. 1997, 29, 214.

36. Freudenberg, K.; Fikentscher H. Liebigs Ann. Chem. 1924, 440, 36. 